OPEN ACCESS

Edited by:

Thomas Egli,

ETH Zürich, Switzerland

Reviewed by:

Lars Regestein,

Leibniz Institute for Natural Product

Research and Infection Biology,

Germany

Sergio Revah,

Autonomous Metropolitan University,

Mexico

*Correspondence:

Kaarel Adamberg

kaarel.adamberg@taltech.ee

Specialty section:

This article was submitted to

Bioprocess Engineering,

a section of the journal

Frontiers in Bioengineering and

Biotechnology

Received: 30 August 2019

Accepted: 13 January 2020

Published: 07 February 2020

Citation:

Adamberg K, Raba G and

Adamberg S (2020) Use

of Changestat for Growth Rate

Studies of Gut Microbiota.

Front. Bioeng. Biotechnol. 8:24. doi: 10.3389/fbioe.2020.00024

\section{Use of Changestat for Growth Rate Studies of Gut Microbiota}

\author{
Kaarel Adamberg ${ }^{1,2 *}$, Grete Raba ${ }^{1}$ and Signe Adamberg ${ }^{1}$ \\ ${ }^{1}$ Department of Chemistry and Biotechnology, Tallinn University of Technology, Tallinn, Estonia, ${ }^{2}$ Center of Food \\ and Fermentation Technologies, Tallinn, Estonia
}

Human colon microbiota, composed of hundreds of different species, is closely associated with several health conditions. Controlled in vitro cultivation and up-todate analytical methods make possible the systematic evaluation of the underlying mechanisms of complex interactions between the members of microbial consortia. Information on reproducing fecal microbial consortia can be used for various clinical and biotechnological applications. In this study, chemostat and changestat cultures were used to elucidate the effects of the physiologically relevant range of dilution rates on the growth and metabolism of adult fecal microbiota. The dilution rate was kept either at $D=0.05$ or $D=0.21 / \mathrm{h}$ in chemostat cultures, while gradually changing from 0.05 to $0.21 / \mathrm{h}$ in the A-stat and from 0.2 to $0.051 / \mathrm{h}$ in the De-stat. Apple pectin as a substrate was used in the chemostat experiments and apple pectin or birch xylan in the changestat experiments, in the presence of porcine mucin in all cases. The analyses were comprised of HPLC for organic acids, UPLC for amino acids, GC for gas composition, 16S-rDNA sequencing for microbial composition, and growth parameter calculations. It was shown that the abundance of most bacterial taxa was determined by the dilution rate on both substrates. Bacteroides ovatus, Bacteroides vulgatus, and Faecalibacterium were prevalent within the whole range of dilution rates. Akkermansia muciniphila and Ruminococcaceae UCG-013 were significantly enriched at $D=0.05$ 1/h, while Bacteroides caccae, Lachnospiraceae unclassified and Escherichia coli clearly preferred $D=0.21 / \mathrm{h}$. In the chemostat cultures, the production of organic acids and gases from pectin was related to the dilution rate. The ratio of acetate, propionate and butyrate was 5:2:1 $(D=0.051 / \mathrm{h})$ and 14:2:1 $(D=0.21 / \mathrm{h})$. It was shown that the growth rate-related characteristics of the fecal microbiota were concise in both directions between $D=0.05$ and $0.21 / \mathrm{h}$. Reproducible adaptation of the fecal microbiota was shown in the continuous culture with a changing dilution rate: changestat. Consortia cultivation is a promising approach for research purposes and several biotechnological applications, including the production of multi-strain probiotics and fecal transplantation mixtures.

Keywords: continuous cultivation, changestat, fecal microbiota, apple pectin, birch xylan

\section{INTRODUCTION}

The cultivation of fecal consortia is essential for understanding the mechanisms behind the coexistence of gut microbial species under changing environmental conditions. This information is required in biotechnological and clinical applications, although better defined methods are required for safer procedures. As the desired consortia can be composed of tens or even hundreds of strains, 
e.g., next generation probiotics, the cultivation of a balanced consortium saves time and labor compared to the production of single cultures. Moreover, the biomass yields of pure cultures can remain below those of natural bacterial communities, because of a deficiency of several growth factors produced by other consortia members. In this field, the culturomics approach has been developed, where a set of different media are used to cultivate each isolate in a multi-parallel approach (Lagier et al., 2012). This approach has expanded the range of microorganisms that can be cultivated in the lab. The reintroduction of fecal microbiota is a promising method to cure certain gastrointestinal conditions (Petrof et al., 2013). Previously, the most diverse stool substitute containing 33 single fecal isolates was developed by Petrof et al. (2013) to cure antibiotic-resistant Clostridium difficile-induced colitis. Also, batch cultures have been used for the production of fecal biomass to treat diarrhea caused by $C$. difficile infection (Jorup-Rönström et al., 2012). Batch cultures are most commonly used for high-throughput small-scale parallel experiments. However, such conditions as substrate concentrations and the accumulation of metabolites are continuously changing. Furthermore, the overgrowth of fast-growing bacteria in mixed cultures is common for batch cultures; for instance, an over $10 \%$ increase in Escherichia coli from the total population has been reported (Brahma et al., 2017; Adamberg et al., 2018). Thus, it is difficult to analyze the actual selectivity of a tested substrate. Consequently, batch technologies are well suited for high throughput screening or for the industrial production of biomass, but have limited value for studies of specific growth mechanisms and the metabolism of complex microbial consortia.

In a microbial consortium, the steady state composition is defined by complex microbial interactions. These interactions can be supportive (mutual or commensal) or inhibitory (ammensal or competitive), and are driven by residual concentrations of substrates, bacterial metabolites and crossfeeding between different bacteria (Gottschal, 1990). By cultivating 37 mouse gut bacteria in continuous mode, Freter et al. (1983) demonstrated that the population dynamics of indigenous intestinal bacteria are controlled by one or a few substrates. Chung et al. (2019) studied the degradation of five different fibers or fiber mixtures by fecal microbiota in a chemostat and showed that mixed fiber substrates led to the growth of more diverse microbiota than inulin alone. In the cultures of gut microbes, cross-feeding has been supposed to be one of the most important factors for gut microbiota richness. To explain the interactions between different microorganisms in a consortium, in addition to cell modeling, simple culture systems, such as defined mixed cultures and single substrates, should be studied first. This makes it possible to elucidate the primary degraders and substrates (hetero- or autotrophies), auxotrophies and compounds derived from cross-feeding. The dynamic data for predicting bacterial behavior in communities can be best obtained from continuous cultures at low substrate concentrations (Russell and Baldwin, 1979; Tannock, 2017). For example, ecological studies have shown that growth under multiple substrate limitations at very low dilution rates supports species having high growth efficiency (higher yield) but low maximal specific growth rate (Gottschal, 1990). Auxotrophy to a specific compound can be used to promote a species in a mixed culture by supplementing the culture medium with this substrate. Two species containing batch experiments revealed that acetate or lactate produced by Bacteroides or Bifidobacterium stimulated the growth of butyric acid producing bacteria, while formate and hydrogen enhanced methanogens (Rowland et al., 2018). The dynamics and stability of freshly collected fecal cultures have been studied by several groups, although the use of fresh samples does not allow for direct comparison of the results from different studies (Miller and Wolin, 1981; Macfarlane et al., 1998; Sghir et al., 1998; McDonald et al., 2013; Yen et al., 2015; Chung et al., 2016; von Martels et al., 2017).

The determination of community composition is essential in microbiota research. Next-generation sequencing methods, such as $16 \mathrm{~S}$ rDNA analysis and whole genome sequencing (WGS), are high throughput approaches that make it possible to identify all of the taxa in whole consortia, but only semi-quantitatively as proportions of the bacteria in a consortium. To obtain quantitative data, bacterial counting through flow cytometry, dry weight analysis or plate counting should be carried out in parallel. Moreover, to determine the taxa of low abundance, the coverage of sequences has to be proportionally higher. Also, species level analysis might require more detailed sequence analysis (WGS) than is available by $16 \mathrm{~S}$ rDNA sequencing. For the quantitative analysis of bacteria in fecal consortia, species are usually assessed by fluorescent in situ hybridization with 16S rRNA probes (Langendijk et al., 1995), although as each species requires a specific probe, this approach is limited by the number of species analyzed or is expensive in an array setup, such as HITChip (Rajilić-Stojanović et al., 2009).

With all cultivation models it should be kept in mind that the specific growth rate of bacteria is not linearly related to the colonic transit rate, since the density of bacteria gradually increases, while the moving rate decreases along the colon. An alternative continuous cultivation technology, changestat, in which all cultivation parameters are computer-controlled, has been developed in our lab. In changestat, the effect of a selected parameter is studied by the gradual change in this parameter within a certain range, while keeping all other conditions constant (Paalme et al., 1995; Kasemets et al., 2003; Adamberg et al., 2015). Our recent study highlighted the importance of dilution rate in determining the composition and diversity of fecal microbiota (Adamberg and Adamberg, 2018). It was also shown that by using de-celerostat (De-stat), the fast- and slowgrowing consortia were differentiated from the fecal microbiota during the same experiment (Adamberg and Adamberg, 2018). To analyze whether these results were too biased depending on the starting point, we carried out experiments in both directions: gradually moving dilution rates from slow to fast (accelerostat: A-stat) and from fast to slow (De-stat), between 0.05 and $0.21 / \mathrm{h}$.

The main aims of the current study were: (1) to elucidate the reproducibility of the chemostat cultures by using the same adult fecal pool, (2) to study the effects of dilution rate on the dynamics and metabolism of fecal microbiota by using A-stat and De-stat cultures, and (3) to elucidate the metabolism of two common dietary fibers, pectin and xylan, by fecal microbiota. Food is an important factor in modulating colonic microbiota and through 
bacterial metabolism promoting health-supporting or diseaseactivating mechanisms. Pectins are a part of the daily diet, consumed in the form of fruits and vegetables and used as food additives. Xylans are abundant in nature as major constituents of hemicellulose in plant cells.

\section{MATERIALS AND METHODS}

\section{Fecal Inoculum}

Fecal samples were collected from seven healthy adult volunteers (19-37 years old, Caucasian, both male and female) and homogenized in four volumes of 5\% DMSO-containing buffer, as described in Adamberg et al. (2015). The exclusion criteria included the use of supplements of prebiotics and probiotics, laxatives and antibiotics for 4 weeks prior to donation. Equal volumes of the seven fecal slurries were pooled together and aliquots were kept at $-80^{\circ} \mathrm{C}$ for repetitive cultivation experiments. Similar sample preparation (standardization by pooling) has also been used and approved by others for in vitro testing in the TIM-2 proximal colon model (Aguirre et al., 2015; Bussolo de Souza et al., 2019).

\section{Defined Base Medium}

The defined growth medium was prepared in a $0.05 \mathrm{M}$ potassium phosphate buffer made from $1 \mathrm{M}$ stock solutions $(\mathrm{ml} / \mathrm{L})$ : $\mathrm{K}_{2} \mathrm{HPO}_{4}$ (28.9) and $\mathrm{KH}_{2} \mathrm{PO}_{4}$ (21.1); mineral salts (mg/L): $\mathrm{MgSO}_{4}{ }^{*} 7 \mathrm{H}_{2} \mathrm{O}(36), \mathrm{FeSO}_{4}{ }^{*} 7 \mathrm{H}_{2} \mathrm{O}(0.1), \mathrm{CaCl}_{2}(9), \mathrm{MnSO}_{4}{ }^{*} \mathrm{H}_{2} \mathrm{O}$ (3), $\mathrm{ZnSO}_{4}{ }^{*} 7 \mathrm{H}_{2} \mathrm{O}$ (1), $\mathrm{CoSO}_{4}{ }^{*} 7 \mathrm{H}_{2} \mathrm{O}$ (1), $\mathrm{CuSO}_{4}{ }^{*} 5 \mathrm{H}_{2} \mathrm{O}$ (1), $\left(\mathrm{NH}_{4}\right)_{6} \mathrm{Mo}_{7} \mathrm{O}_{24} * 4 \mathrm{H}_{2} \mathrm{O}(1), \mathrm{NaCl}(527)$; hemin (5 mg/L); vitamin K1 (0.5 mg/L); L-amino acids (g/L): Ala (0.044), Arg (0.023), Asn (0.038), Asp (0.038), Glu (0.036), Gln (0.018), Gly (0.032), His (0.027), Ile (0.060), Leu (0.120), Lys-HCl (0.080), Met (0.023), Phe (0.050), Pro (0.041), Ser (0.095), Thr (0.041), Trp (0.009), Val (0.060), Tyr (0.015); vitamins (mg/L): biotin (0.25), Ca-pantothenate $(0.25)$, folic acid (0.25), nicotinamide $(0.25)$, pyridoxine- $\mathrm{HCl}(0.50)$, riboflavin $(0.25)$, thiamine- $\mathrm{HCl}(0.25)$ and other components (g/L): bile salts (0.5), $\mathrm{NaHCO}_{3}(2.0)$, Tween-80 (0.5), Na-thioglycolate (0.5), and Cys- $\mathrm{HCl}$ (0.5, freshly made). The carbohydrate substrates were sterilized separately and mixed with the medium before cultivation. Two substrate combinations, either birch xylan (Sigma-Aldrich, United States) or apple pectin (Sigma-Aldrich, United States) with porcine mucin (Type II, Sigma Aldrich, United States), were added to the base medium in equal amounts $(2.5 \mathrm{~g} / \mathrm{L}$ each). The $\mathrm{pH}$ of the growth medium was $7.2 \pm 0.1$.

\section{Fermentation System}

The Biobundle cultivation system consisted of the ADI 1030 bio-controller and cultivation control program "BioXpert" (Applikon, Netherlands). The fermenter was equipped with sensors for $\mathrm{pH}, \mathrm{pO}_{2}$, and temperature. Variable speed pumps for feeding and outflow were controlled by a De-stat or A-stat algorithm: $\mathrm{D}=\mathrm{D}_{0}-\mathrm{d}^{*} \mathrm{t}$ or $\mathrm{D}=\mathrm{D}_{0}+\mathrm{a}^{*} \mathrm{t}$, respectively, where $\mathrm{D}$ is the dilution rate $(1 / \mathrm{h}), \mathrm{D}_{0}$ is the initial dilution rate, $\mathrm{d}$ and a are the deceleration and acceleration rate $\left(1 / h^{2}\right)$, respectively, and $t$ is the time $(\mathrm{h})$. In accelerostat (A-stat), the dilution rate was gradually increased from 0.05 to $0.21 / \mathrm{h}$ and in decelerostat (De-stat) the dilution rate was gradually decreased from 0.2 to $0.05 \mathrm{l} / \mathrm{h}$ in accordance with the typical transit rate of the human colon (Adamberg and Adamberg, 2018). pH was controlled by a $1 \mathrm{M} \mathrm{NaOH}$ addition according to the $\mathrm{pH}$ set-point. The culture volume was kept constant $(300 \mathrm{~mL})$ by monitoring the weight of the fermenter with the PC-linked balance and outflow pump. The $\mathrm{pH}$ of the culture was kept at 7.0 and the temperature was kept constant at $36.6^{\circ} \mathrm{C}$. The medium in the feeding bottle and the fermenter was flushed with sterile-filtered nitrogen gas (99.9\%, AGA) overnight before inoculation and throughout the cultivation to maintain anaerobiosis. Nitrogen flushing was on during the whole experiment. Two $\mathrm{mL}$ of the pooled fecal culture was inoculated to start the experiment.

The cultivation algorithm was started $15-17 \mathrm{~h}$ after inoculation in the midst of the exponential growth of bacteria. The dilution rate was stabilized at either 0.05 or $0.21 / \mathrm{h}$, at $\mathrm{pH}$ 7.0, and run for stabilization at these conditions by 6-7 residence times. After achieving a stable titration rate and gas production, the dilution rate was decreased to $0.051 /$ h or increased to $0.21 / \mathrm{h}$ at a rate of 0.05 units per day (the experimental timeline is presented in Figure 1). The dilution rate interval was chosen to cover the realistic growth rates of luminal bacteria in the colon. The range of the specific growth rate of the bacteria was calculated based on the colonic transit time of digesta in people consuming Western diets, which varies from 40 to $140 \mathrm{~h}$ (median 60-70 h) (Burkitt et al., 1972; Cummings et al., 1976; Fallingborg et al., 1989), and the estimated amount of bacteria, which increases from $10^{8}$ in the proximal colon to $10^{11} \mathrm{cfu} / \mathrm{g}$ in feces (Sender et al., 2016). Considering both the period the bacteria have for degradation of dietary fibers and the coinciding increase in the bacterial biomass in the colon, the specific growth rate of the bacteria decreased from 0.3 to $0.02 \mathrm{1} / \mathrm{h}$. Thus, the range of the dilution rates tested in the cultivation experiments, $0.05-0.21 / \mathrm{h}$, was chosen.

\section{Analytical Methods}

Samples from the outflow were collected on ice, centrifuged $\left(14,000 \mathrm{~g}, 5 \mathrm{~min}, 4^{\circ} \mathrm{C}\right)$ and stored separately as pellets and supernatants at $-20^{\circ} \mathrm{C}$ until HPLC analyses (sugars and organic acids), UPLC analyses (amino acids), and microbial 16S rDNA sequencing were carried out.

For chromatographic analyses, culture supernatants were filtered using AmiconR Ultra-10K Centrifugal Filter Devices, cut-off $3 \mathrm{kDa}$ according to the manufacturer's instructions (Millipore, United States). The concentrations of organic acids (succinate, lactate, formate, acetate, propionate, isobutyrate, butyrate, isovalerate, and valerate), ethanol and free sugars (mono-, di-, and trisaccharides) were determined by highperformance liquid chromatography (HPLC, Alliance 2795 system, Waters, Milford, MA, United States), using BioRad HPX$87 \mathrm{H}$ column (Hercules, CA, United States) with isocratic elution of $0.005 \mathrm{M} \mathrm{H}_{2} \mathrm{SO}_{4}$ at a flow rate of $0.5 \mathrm{~mL} / \mathrm{min}$ and at $35^{\circ} \mathrm{C}$. Refractive index (RI) (model 2414; Waters, United States) and UV (210 nm; model 2487; Waters, United States) detectors were used for quantification of the substances. The detection limit for the HPLC method was $0.1 \mathrm{mM}$. Concentrations of 


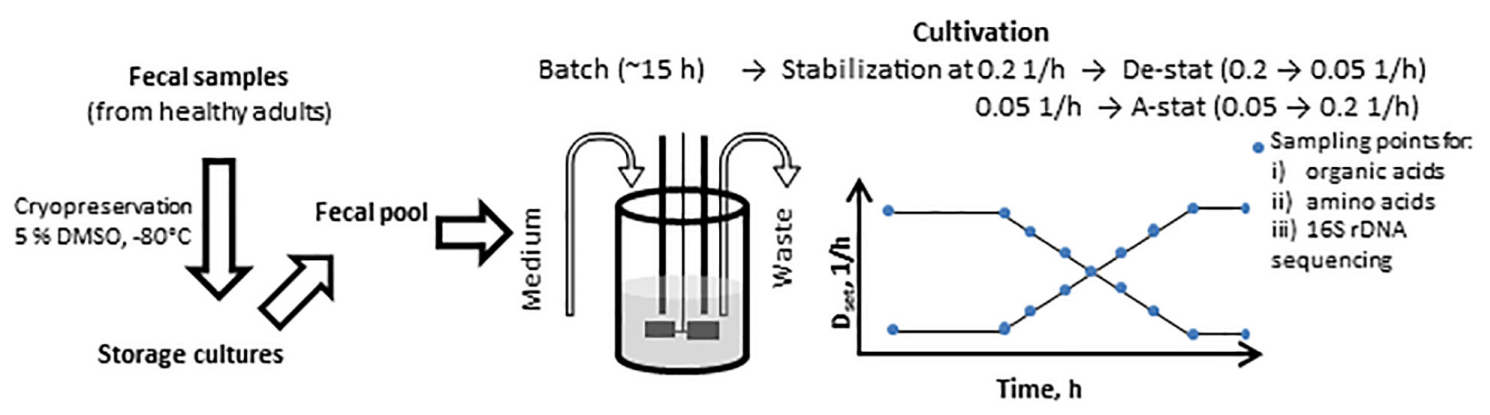

FIGURE 1 | Scheme of the A-stat (accelerostat) and De-stat (decelerostat) experimental set-up. The pooled fecal inoculum was added into $300 \mathrm{ml}$ growth medium at time $0 \mathrm{~h}$ followed by batch phase (ca $15 \mathrm{~h}$ ) until mid- exponential growth phase. Then continuous mode was started and after the stabilization of the fecal culture at $D=0.21 / \mathrm{h}$ (De-stat) or $D=0.051 / \mathrm{h}$ (A-stat, $6-7$ residential times in total), the dilution rate was gradually decreased down to $0.051 / \mathrm{h}$ or increased up to $0.21 / \mathrm{h}$ (deceleration or acceleration rate $0.051 / \mathrm{h}$ per day), respectively, followed by re-stabilization for approximately 2 residential times. The same procedure was applied

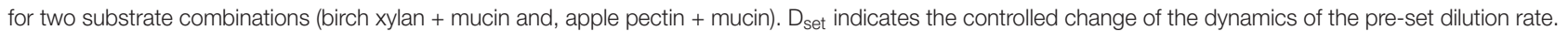

amino acids and amines were determined with an amino acid analyzer (UPLC; Waters, Milford, United States) according to the manufacturer's instructions. The detection limit of the method was $0.01 \mathrm{mM}$. All standard substrates were of analytical grade. Empower software (Waters, United States) was used for the processing of HPLC and UPLC data.

The composition of the gas outflow $\left(\mathrm{H}_{2}, \mathrm{CO}_{2}, \mathrm{H}_{2} \mathrm{~S}, \mathrm{CH}_{4}\right.$, and $\mathrm{N}_{2}$ ) was analyzed using an Agilent 490 Micro GC Biogas Analyzer (Agilent 269 Technologies Ltd., United States) connected to a thermal conductivity detector. The volume of the gas flow was regularly recorded.

The Redox potential of the growth medium and culture supernatant was measured by a $\mathrm{pH} /$ Redox meter using an InLab ${ }^{\circledR}$ Redox electrode (Mettler Toledo).

The biomass dry weight was measured gravimetrically by centrifuging the biomass from a $10 \mathrm{~mL}$ culture, washing twice with distilled water and drying in an oven at $105^{\circ} \mathrm{C}$ for $24 \mathrm{~h}$.

\section{DNA Extraction and Amplification}

DNA was extracted from the pellets using a PureLink Microbiome DNA extraction kit (Thermo Fisher Scientific, United Kingdom) according to the manufacturer's instructions. Universal primers:

S-D-Bact-0341-b-S-17 Forward (5'TCGTCGGCAGCGTCAG ATGTGTATAAGAGACAGCCTACGGGNGGCWGCAG) and S-D-Bact-0785-a-A-21 Reverse (5'GTCTCGTGGGCTCGGA GATGTGTATAAGAGACAGGACTACHVGGGTATCTAATCC) were used for PCR amplification of the V3-V4 hypervariable regions of the 16S rRNA genes (Klindworth et al., 2013). The amplified region was 390-410 bp long and an average of 67,000 reads per sample were obtained. The mixture of amplicons was sequenced using an Illumina MiSeq $2 \times 250$ v2 platform (Estonian Genome Centre, University of Tartu, Estonia).

\section{Taxonomic Profiling of Microbiota Samples}

The DNA sequence data was analyzed using a BION-meta ${ }^{1}$, currently unpublished open source program, according to the

${ }^{1}$ www.box.com/bion author's instructions. Sequences were first cleaned at both ends using a $99.5 \%$ minimum quality threshold for at least 18 of 20 bases for $5^{\prime}$-end and 28 of 30 bases for $3^{\prime}$-end, then joined, followed by the removal of contigs shorter than $350 \mathrm{bp}$. Then sequences were cleaned of chimeras and clustered by 95\% oligonucleotide similarity (k-mer length of $8 \mathrm{bp}$, step size $2 \mathrm{bp}$ ). Lastly, consensus reads were aligned to the SILVA reference 16S rDNA database (v123) using a word length of 8 and similarity cut-off of $90 \%$. The bacterial designation was analyzed at different taxonomic levels, down to species if applicable.

\section{Calculations}

For quantitative data analysis, the relative data of bacterial abundances from $16 \mathrm{~S}$ rDNA sequencing analysis were first converted to quantitative values $\left[\mathrm{X}_{\mathrm{i}}(\mathrm{g} / \mathrm{L})\right.$, where $\mathrm{i}$ illustrates bacterial taxa i] by the formula: $\mathrm{X}_{\mathrm{i}}=\mathrm{X}_{\mathrm{t}}{ }^{*} \mathrm{~A}_{\mathrm{i}}$, where $\mathrm{X}_{\mathrm{t}}$ is the dry weight of the total biomass of bacteria $(\mathrm{g} / \mathrm{L})$ and $\mathrm{A}_{\mathrm{i}}$ is the relative abundance of bacterial taxa $i$ in the sample.

The growth characteristics of the bacteria in A-stat and De-stat experiments were calculated based on bacterial mass, total volume of medium pumped out from the fermenter ( $V_{\text {OUT }}$, $\mathrm{L})$ and product concentrations in the culture medium $(\mathrm{mol} / \mathrm{L})$ as follows:

$$
\begin{gathered}
\mu=\frac{d\left(V_{\text {OUT }}\right)}{V \times d t}+\frac{d\left(X_{t}\right)}{d t \times X_{t}} \\
Q_{S i}=\frac{S_{i} \times d\left(V_{\text {OUT }}\right)}{V \times X_{t} \times d t}-\frac{d\left(S_{i}\right)}{d t \times X_{t}} \\
Q_{P i}=\frac{P_{i} \times d\left(V_{O U T}\right)}{V \times X_{t} \times d t}+\frac{d\left(P_{i}\right)}{d t \times X_{t}}
\end{gathered}
$$

where $\mu$ is the specific growth rate $(1 / \mathrm{h}), Q_{S}$ is the specific consumption rate of carbohydrate (in carbon equivalents, mol$\left.\mathrm{C} / \mathrm{g}-\mathrm{X}_{\mathrm{t}} / \mathrm{h}\right), \mathrm{S}_{\mathrm{i}}$ is the concentration of consumed carbohydrate $\mathrm{i}$ $(\mathrm{C}-\mathrm{mol} / \mathrm{L}), \mathrm{Q}_{\mathrm{Pi}}$ is the specific production rate of product $\mathrm{i}$ (mol$\operatorname{prod} / \mathrm{g}-\mathrm{X} / \mathrm{h}), \mathrm{P}_{\mathrm{i}}$ is the concentration of product $\mathrm{i}(\mathrm{mol} / \mathrm{L}), \mathrm{V}$ is the current fermenter volume $(\mathrm{L}), \mathrm{V}_{\text {OUT }}$ is the outflow volume and $\mathrm{t}$ is the cultivation time $(\mathrm{h})$. 


\section{Statistical Analysis}

Concentrations of metabolites or abundances of bacteria from three independent experiments were compared by average values and by unpaired and t-test (unadjusted $P$-values $<0.05$ were considered significant) for chemostat point comparison. To compare differences in bacterial abundances and metabolite productions during A-stat and De-stat experiments, samples were divided into two groups: (1) samples taken at $D<0.071 / \mathrm{h}$ (slow growth) and (2) samples taken at $D>0.171 / \mathrm{h}$ (fast growth). Average and standard deviation of bacterial abundances and metabolite productions were calculated in both groups and a single parametric t-test was used to estimate statistical significance.

\section{Ethics Statement}

This study was approved by the Tallinn Medical Research Ethics Committee, Estonia (protocol no. 554).

\section{RESULTS}

\section{Reproducibility of Chemostat Cultures of Fecal Microbiota}

Until now, continuous fecal cultures have mostly been inoculated with fresh fecal inocula and information on reproducibility of this type of experiment is scarce. Hence, the aim of this work was to elucidate the reproducibility of the continuous fecal cultures. To elucidate the reproducibility of the growth of biological replicates of adult pooled fecal microbiota, six chemostat cultures (three at $\mathrm{D}_{\text {low }}=0.051 / \mathrm{h}$ and three at $\mathrm{D}_{\text {high }}=0.21 / \mathrm{h}$ ) in a defined base medium with apple pectin and mucin were carried out.

\section{Formation of Organic Acids and Gases}

The production of organic acids and gases from pectin and mucin was related to the specific growth rate (Figure 2). The most abundant organic acid in all chemostat cultures was acetate $\left[23.2 \pm 3.1\right.$ and $25.2 \pm 2.0 \mathrm{mM}$ at $\mathrm{D}_{\text {low }}(0.051 / \mathrm{h})$ and $D_{\text {high }}(0.21 / h)$, respectively]. Compared to $D_{\text {high, the }}$ production of propionate, butyrate and carbon dioxide was more enhanced at $\mathrm{D}_{\text {low. }}$. At $\mathrm{D}_{\text {low }}, 9.4 \pm 0.3 \mathrm{mM}$ propionate and less than $4 \mathrm{mM}$ succinate were produced, while at $\mathrm{D}_{\text {high }}$, concentrations of propionate and succinate were practically equal (4.2 \pm 0.3 and $3.9 \pm 0.8 \mathrm{mM}$, respectively). The ratio of acetate, propionate and butyrate was 5:2:1 at $\mathrm{D}_{\text {low }}$ and 14:2:1 at $\mathrm{D}_{\text {high. }}$. Similar to the product profile at $\mathrm{D}_{\text {low }}$, a ratio of $4: 2: 1$ for acetate:propionate:butyrate was reported by Larsen et al. (2019) in TIM-2 experiments. In accordance with the organic acid profiles, about twice as much carbon dioxide was produced at $\mathrm{D}_{\text {low }}$ than at $\mathrm{D}_{\text {high }}(20.3 \pm 2.4$ and $10.5 \pm 2.8 \mathrm{mmol}$ per $\mathrm{L}$ medium, respectively). On average, the relative difference between parallels of the concentrations of propionate, butyrate and lactate in stabilized fecal cultures (after six residential times) remained below $10 \%$ and that of acetate below $25 \%$ at both dilution rates $\left(\mathrm{D}_{\text {low }}\right.$ and $\left.\mathrm{D}_{\text {high }}\right)$.

In addition to organic acids and gases, the consumption of amino acids was analyzed. It was determined that amino

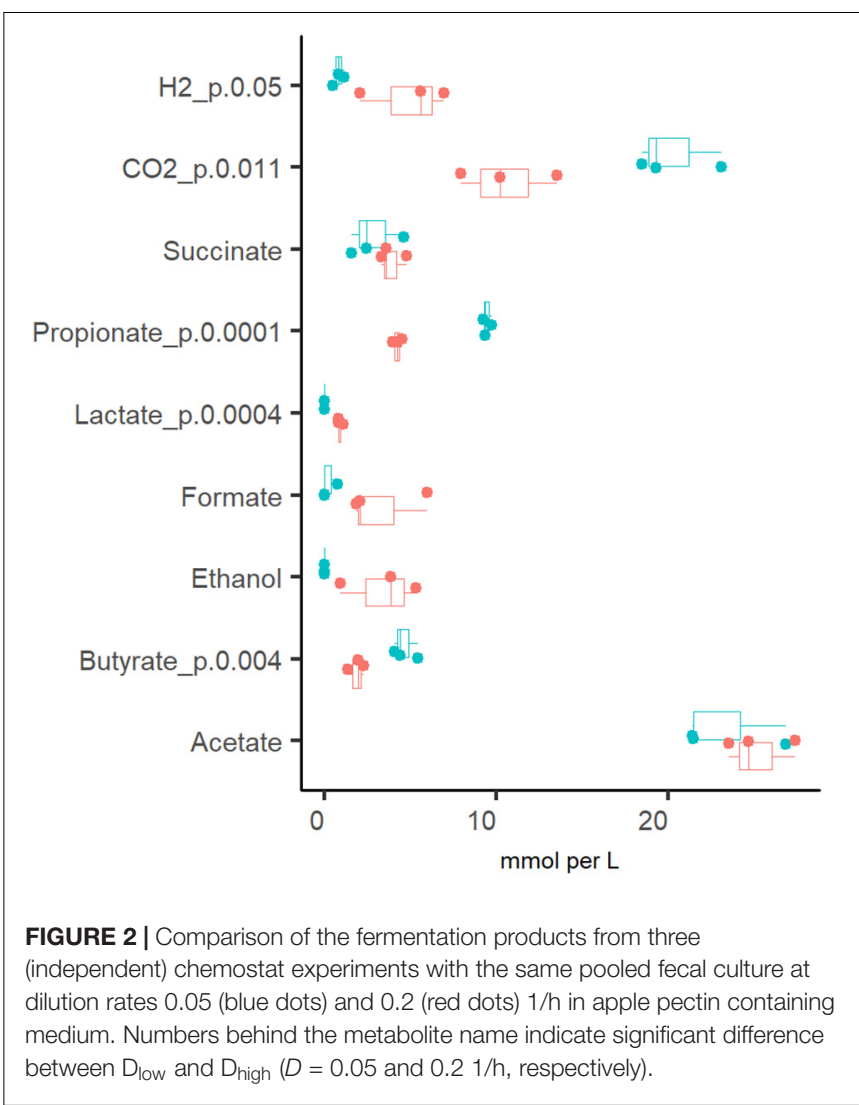

acids were fully depleted from the culture medium at both dilution rates, except for alanine and branched-chain amino acids (BCAA), which were practically not consumed at $\mathrm{D}_{\text {high }}$ (Figure 3 ). The increased production of propionate, butyrate and $\mathrm{CO}_{2}$ at $\mathrm{D}_{\text {low }}$ was accompanied by the conversion of BCAA to isobutyric $(0.27 \pm 0.29 \mathrm{mM})$ and isovaleric acids $(0.87 \pm 0.82 \mathrm{mM})$. Another amino acid degradation product significantly higher at $\mathrm{D}_{\text {low }}$ was $\mathrm{H}_{2} \mathrm{~S}(0.76 \pm 0.12$ vs. $0.36 \pm 0.09 \mathrm{mmol}$ per $\mathrm{L}$ medium at $\mathrm{D}_{\text {low }}$ vs. $\mathrm{D}_{\text {high }}$, respectively) derived from the sulfur-containing amino acids Cys and Met. At $\mathrm{D}_{\text {high }}$, isoleucine and leucine were consumed in the range required for biomass formation (0.18-0.24 and 0.45-0.58 $\mathrm{mmol} / \mathrm{gDW}$, respectively. Supplementary Table S2 Chemostat), based on the amino acid contents in the biomass of E. coli ( 0.22 and $0.37 \mathrm{mmol} / \mathrm{gDW}$ for Ile and Leu, respectively; Valgepea et al., 2011) and Lactococcus lactis (0.25 and $0.37 \mathrm{mmol} / \mathrm{gDW}$ for Ile and Leu, respectively; Adamberg et al., 2012). As the total amount of biomass produced was $0.5-0.7 \mathrm{~g} / \mathrm{L}$ (Supplementary Table S2 Chemostat), the consumption of other amino acids exceeded 1.6-5.5 times the amount required for biomass synthesis, except for serine, which was consumed about 10 times as much. Serine is the major amino acid in mucins and may be converted to acetate. However, serine degradation $\left(2.1\right.$ and $2.6 \mathrm{mmol} / \mathrm{gDW}$ at $\mathrm{D}_{\text {low }}$ and $\mathrm{D}_{\text {high }}$, respectively) could not have contributed to more than $5 \%$ of the total acetate production (39 and $51 \mathrm{mmol} / \mathrm{gDW}$ at $\mathrm{D}_{\text {low }}$ vs. $\mathrm{D}_{\text {high }}$, respectively) as $80-86 \%$ of the carbon was derived 


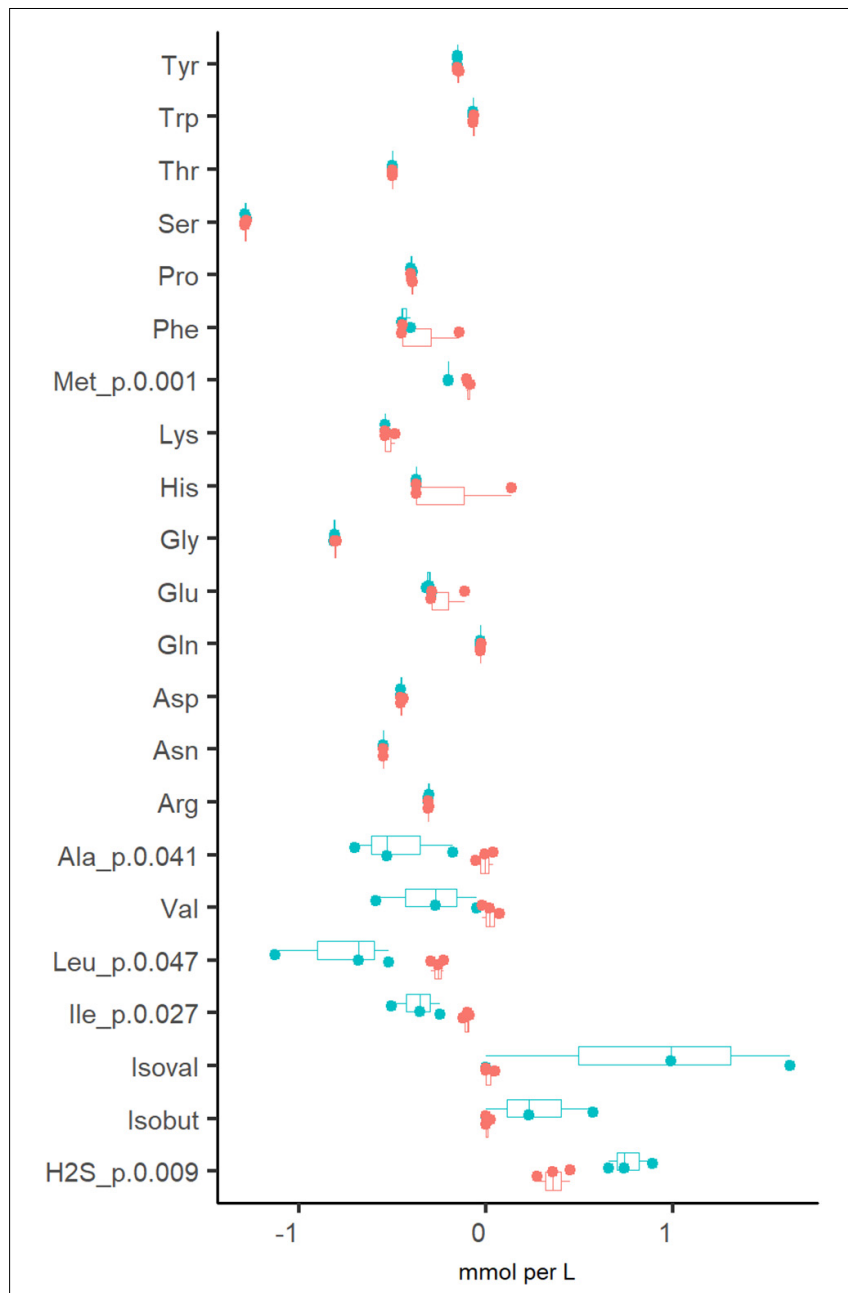

FIGURE 3 | Comparison of amino acid consumptions and related metabolites (branched chain fatty acids and $\mathrm{H}_{2} \mathrm{~S}$ ) from three independent chemostat experiments of fecal culture at dilution rate 0.05 (blue dots) and 0.2 (red dots) $1 / \mathrm{h}$ in apple pectin medium. Positive values indicate the production and negative values indicate the consumption of the amino acid. Numbers behind the metabolite name indicate significant difference between $D_{\text {low }}$ and $D_{\text {high }}$ ( $D=0.05$ and $0.21 / \mathrm{h}$, respectively). isoval, isovalerate; isobut, isobutyrate.

from carbohydrate fermentation. The overall carbon recovery was $79 \%$ and $67 \%$ at $D_{\text {low }}$ and $D_{\text {high }}$, respectively, showing that some products were under-determined or missing, especially at high dilution rate.

\section{Growth Rate Specific Differences of Fecal Microbiota} The profiles of the metabolic products were in accordance with the bacterial compositions detected (Figures 2-4). Three taxa clearly prevalent at both dilution rates were the acetate- and propionate- or succinate-producing species Bacteroides ovatus (17 and $14 \%$, at $\mathrm{D}_{\text {low }}$ and $\mathrm{D}_{\text {high }}$, respectively), Bacteroides vulgatus (7.9 and $3.6 \%$, at $\mathrm{D}_{\text {low }}$ and $\mathrm{D}_{\text {high }}$, respectively) and butyrateproducing bacterium Faecalibacterium (2.4 and 7.2\%, at $\mathrm{D}_{\text {low }}$ and $\mathrm{D}_{\text {high }}$, respectively). Also several other bacteria were abundant (1-4\% of the total population) at both dilution rates, such as mixed acid (acetate, propionate and butyrate) fermenting

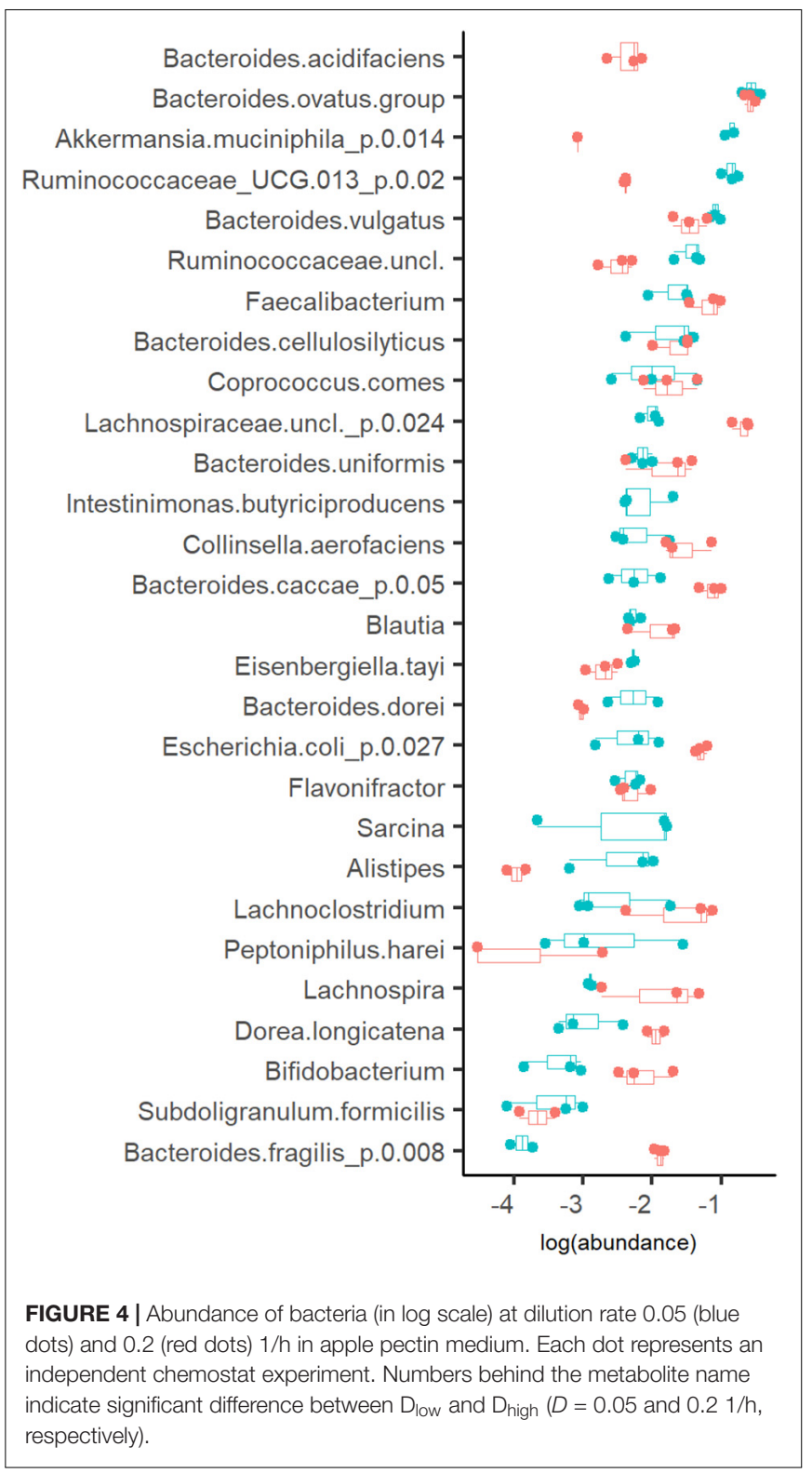

bacteria, and the acetate- and propionate-producing Bacteroides uniformis and Bacteroides cellulosilyticus (Figure 4). At $\mathrm{D}_{\text {low }}$ the mucin degrading species Akkermansia muciniphila and a group of Ruminococcaceae UCG-013 (from 0.1 to $16 \%$ and from 0.5 to $14 \%$, respectively) were significantly enriched. In different, at $\mathrm{D}_{\text {high }}$, Bacteroides caccae, Lachnospiraceae unclassified and mainly acetate-producing E. coli $(7.7 \%, 21 \%$ and $6.3 \%$ of total reads, respectively) became dominant. The butyrate-producing bacterium Intestimonas butyriciproducens and Sarcina were detected only at $\mathrm{D}_{\text {low }}$, whereas Bacteroides acidifaciens was found only at $\mathrm{D}_{\text {high }}$. The increased production of ethanol and formate at $\mathrm{D}_{\text {high }}$ can be linked to higher abundances of Dorea and Blautia.

A remarkable enrichment of Enterobacteriaceae (up to $60 \%$ of total population) was observed in batch phase before starting the 
continuous flow. The amount of E. coli formed nearly $50 \%$ of the microbial population but decreased to about $10 \%$ in a chemostat stabilized at $\mathrm{D}_{\text {high }}$, and to $1.2 \%$ at $\mathrm{D}_{\text {low }}$. These data confirm the competitiveness of the fast-growing $E$. coli at high dilution rates.

\section{Comparison of A-Stat and De-Stat Cultures}

Changes in the dilution rate in both directions between 0.05 to $0.2 \mathrm{l} / \mathrm{h}$, starting from the stabilized cultures of pooled fecal microbiota in apple pectin and birch xylan media, were analyzed. In A-stat, the dilution rate was gradually increased from 0.05 to $0.2 \mathrm{l} / \mathrm{h}$, and in De-stat the dilution rate was gradually decreased from 0.2 to $0.05 \mathrm{l} / \mathrm{h}$. The stabilization of the chemostat culture was controlled by the titration rate of sodium hydroxide, indicating the rate of acid production rate, and the gas production rate (Supplementary Figure S1). Average fluctuations of these parameters below 5\% within the last three residential times were considered to be stable cultures to start the changestat algorithm (on average, six or seven residential volumes were needed to achieve the stable cultures).

\section{Formation of Organic Acids and Gases}

In total, of one mole of carbohydrates, $1.2-1.3$ and $1.7-1.9 \mathrm{~mol}$ of acids were produced in xylan and pectin supplemented media, respectively. Acetate formed in nearly two thirds of all fermentation products and its production did not depend on the dilution rate (Figure 5). Except for acetate and carbon dioxide, the formation of other metabolites from pectin and xylan was comparable in both directions of the dilution rate change (from 0.05 to 0.2 and from 0.2 to $0.051 / \mathrm{h}$ ). Almost twice as much acetate was produced from xylan than from pectin (0.6-0.7 and 1.1$1.2 \mathrm{~mol}$ per mole of carbohydrates consumed, respectively). As xylose is a five-carbon sugar and galacturonic acid is a six-carbon compound, the lower amount of acids produced in the xylan medium can partly be explained by these differences. However, at all dilution rates, the carbon balance $\left(\mathrm{C}_{\text {substrates }}-\mathrm{C}_{\text {products }}\right)$ was still lower in the xylan- than in the pectin-containing medium (average values $74 \pm 5 \%$ and $82 \pm 2 \%$, respectively). The formation of other metabolites, especially carbon dioxide and propionate, was strongly dilution rate-dependent. The synthesis of carbon dioxide was 0.9 and $0.4 \mathrm{mmol}$ per $\mathrm{L}$ medium at $\mathrm{D}_{\text {low }}$ and at $\mathrm{D}_{\text {high }}$ in the pectin medium in both experimental directions (A-stat and De-stat). A similar trend was observed for xylan, suggesting that the production of carbon dioxide is linked to the growth rate rather than the substrate. To compensate for the change in the carbon flux caused by the decreased production of $\mathrm{CO}_{2}$, the succinate production increased, especially in the xylan medium. For example, in the A-stat experiment of the xylan medium, the reduction of $\mathrm{CO}_{2}$ production from 0.42 to $0.22 \mathrm{~mol}$ per mole of carbohydrates was compensated for by enhanced formation of succinate $(0.26$ to 0.42 at $D=0.051 / \mathrm{h}$ and $D=0.21 / \mathrm{h}$, respectively) and formate (0.02 to $0.2 \mathrm{~mol}$ per mol carbohydrates at $D=0.051 / \mathrm{h}$ and $D=0.21 / \mathrm{h}$, respectively). Similarly, propionate synthesis was decreased as a response to increasing formate production, keeping the carbon flux consistent $(0.27$ to $0.09 \mathrm{~mol}$ per mol carbohydrates at $D=0.051 / \mathrm{h}$ and $D=0.21 / \mathrm{h}$, respectively). A reverse correlation between concentrations of propionic and succinic acids was observed. In comparing the gas production, notably less carbon dioxide (0.3-0.4 mol per mole of carbohydrates consumed) was formed from xylan, built of xylose, a five-carbon molecule, at all dilution rates. Carbon dioxide may originate from succinate to propionate conversion or butyrate production or demetoxylation of metoxylated galacturonic acid (Figure 5).

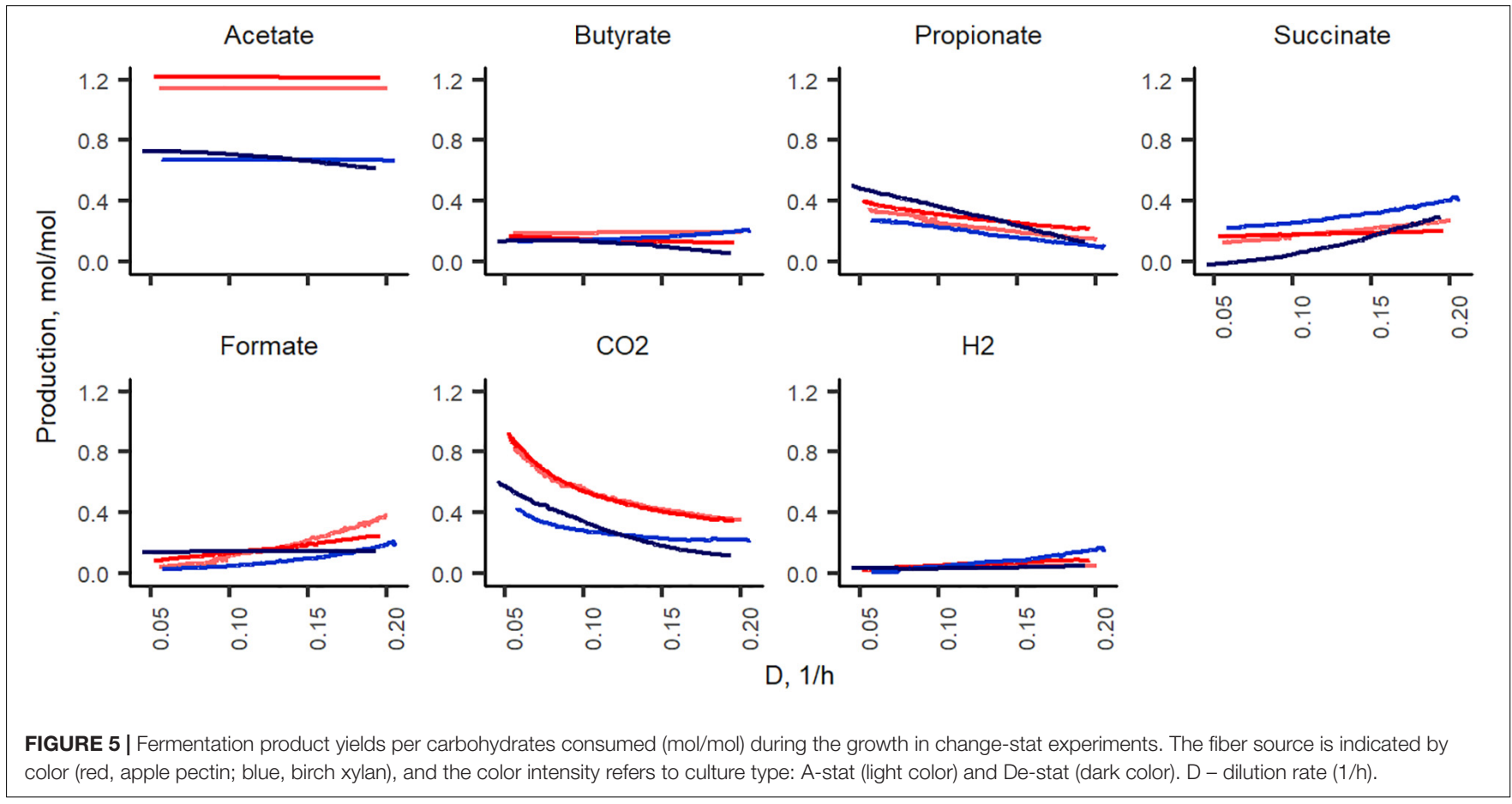


The described changes were characteristic under both directions of the dilution rate (A-stat and De-stat). These data suggest that the acceleration rate applied allowed the culture to adapt to the changing conditions.

\section{Consumption and Formation of Amino Acids}

Similarly to the chemostat cultures, most of the amino acids were completely depleted from the medium, except for alanine and BCAA (Table 1). The consumption of valine and leucine increased at lower dilution rates. The formation of isobutyrate and isovalerate was practically missing at high dilution rates, but they were produced in concentrations of 2.2-11 and 13-44 $\mathrm{mmol} / \mathrm{mol}$-carbohydrates, respectively, at low dilution rates $(D<1 / \mathrm{h})$ (Table 1$)$. In both media, the alanine metabolism was more intensive at low dilution rates (up to $14 \mathrm{mmol} / \mathrm{mol}$-carbohydrates). The degradation of the reducing agent cysteine to $\mathrm{H}_{2} \mathrm{~S}$ up to $41 \mathrm{mmol} / \mathrm{mol}$ carbohydrates was observed at low dilution rates in all experiments (Table 1), which corresponds to the degradation of $22 \%$ of the total cysteine.

\section{Growth Rate Specific Changes in Fecal Microbiota}

The initial fecal slurry contained 88 bacterial species with abundance above $0.1 \%$ and, of these, 25 species had abundance higher than 1\% (Supplementary Table S1). During the chemostat and the following A-stat and De-stat cultivations, the species richness decreased to $27-32$ and 10-18 species with abundance of 0.1 and $1 \%$ with apple pectin and xylan, respectively. A significant decrease in species richness has been shown by other authors (McDonald et al., 2013; Chung et al., 2016). The abundance of the majority of bacterial taxa was determined by the dilution rate on both substrates. The prevailing genus in the consortia - Bacteroides (up to 58\% of the total population) - adapted well within the whole range of specific growth rates tested. However, the abundances of some species, such as B. ovatus and Bacteroides cellulosilyticus, tended to decrease at higher dilution rates (A-stat) in the xylansupplemented medium ( $p=0.02$ ) (Figure 6). Pectin selectively enriched the Ruminococcaceae group UCG013, which was never detected in the xylan-containing medium. The abundance of the Ruminococcaceae group UCG-013 was also related to the dilution rate being $17 \%$ at lower dilution rates and down to $5 \%$ at dilution rates below $D<0.15$ in both change directions (Figure 6). These data are in accordance with the chemostat results.

Significant increases in abundances of Collinsella aerofaciens $(p=0.002)$, E. coli $(p=0.001)$, Faecalibacterium prausnitzii $(p=0.009)$ and a group of Lachnospiraceae (closest similarity to Coprococcus, $p=0.008$ ) (median abundances 1.6, 12, 5.1, and $19 \%$, respectively) were observed at dilution rates above $0.171 / \mathrm{h}$ on both substrates. Although the abundances of butyrate-producing bacteria (Faecalibacterium, Coprococcus, and Lachnospiraceae) increased along with the increasing dilution rate, the tested substrates and conditions did not enhance the production of butyrate, resulting in other fermentation products instead.

At higher dilution rates, the increased formate production was accompanied by higher amounts of species from the genus Lachnoclostridium that are known to be involved in formate production. The abundance of the prevailing species at dilution rates below 0.07 1/h, A. muciniphila (median abundance 22\%) decreased significantly at dilution rates above $0.171 / \mathrm{h}$ (abundance $>1 \%$ ) in all changestat experiments. This is in accordance with the production of propionic acid, the characteristic metabolite of Akkermansia (Figure 5). Another taxa inhibited at higher dilution rates was Intestinimonas (Figure 6).

\section{DISCUSSION}

In continuous cultures, the environmental parameters, including substrate concentrations, $\mathrm{pH}$, and flow rate, can be precisely controlled. The in vivo situation in the colon probably remains somewhere between chemostat and batch states, i.e., the availability of fermentable substrates decreases, the amounts of metabolites change dynamically and $\mathrm{pH}$ moves toward the alkaline region slowly. Our results demonstrate that the changestat techniques, the A-stat and De-stat, can be applied to study the effects of growth rate on the composition and metabolism of fecal microbiota. Using the same fecal inoculum, we showed that continuous cultures are reproducible at dilution rates of $D=0.2$ and $0.051 / \mathrm{h}$. The dilution rate during the stabilization phase impacts the results of

TABLE 1 | Consumption of amino acids and formation of degradation products from amino acids (mmol per mol carbohydrates consumed) during A-stat and De-stat experiments and significantly different at fast or slow dilution rate.

\begin{tabular}{|c|c|c|c|c|c|c|c|c|}
\hline Experiment* & State ${ }^{\star \star}$ & $D, 1 / h$ & Isobutyrate & Isovalerate & $\mathrm{H}_{2} \mathrm{~S}$ & Ala & Ile & Leu \\
\hline Xyl_A & SS & 0.058 & 2.2 & 13.6 & 22.1 & 11.0 & 6.4 & 12.7 \\
\hline Xyl_D & $Q$ & 0.058 & ND & ND & 29.7 & 2.3 & 4.8 & 12.6 \\
\hline Xyl_D & SS & 0.193 & ND & 23.9 & 7.4 & ND & 2.8 & 6.8 \\
\hline Xyl_A & $Q$ & 0.193 & ND & 1.0 & 12.4 & 3.4 & 4.1 & 5.5 \\
\hline Pec_A & SS & 0.055 & 11.3 & 44.1 & 29.1 & 6.9 & 10.6 & 16.3 \\
\hline Pec_D & $Q$ & 0.054 & 2.7 & 17.1 & 41.3 & 14.3 & 8.8 & 15.2 \\
\hline Pec_D & SS & 0.196 & 0.2 & 1.8 & 12.2 & ND & 5.7 & 8.5 \\
\hline Pec_A & $Q$ & 0.197 & 4.1 & 12.2 & 16.0 & ND & 5.4 & 2.2 \\
\hline
\end{tabular}

${ }^{*} A$ and $D$ indicate A-stat and De-stat, respectively. ${ }^{* *} S S$ and $Q$ indicate steady state and quasi steady state, respectively. ND, not detected. 


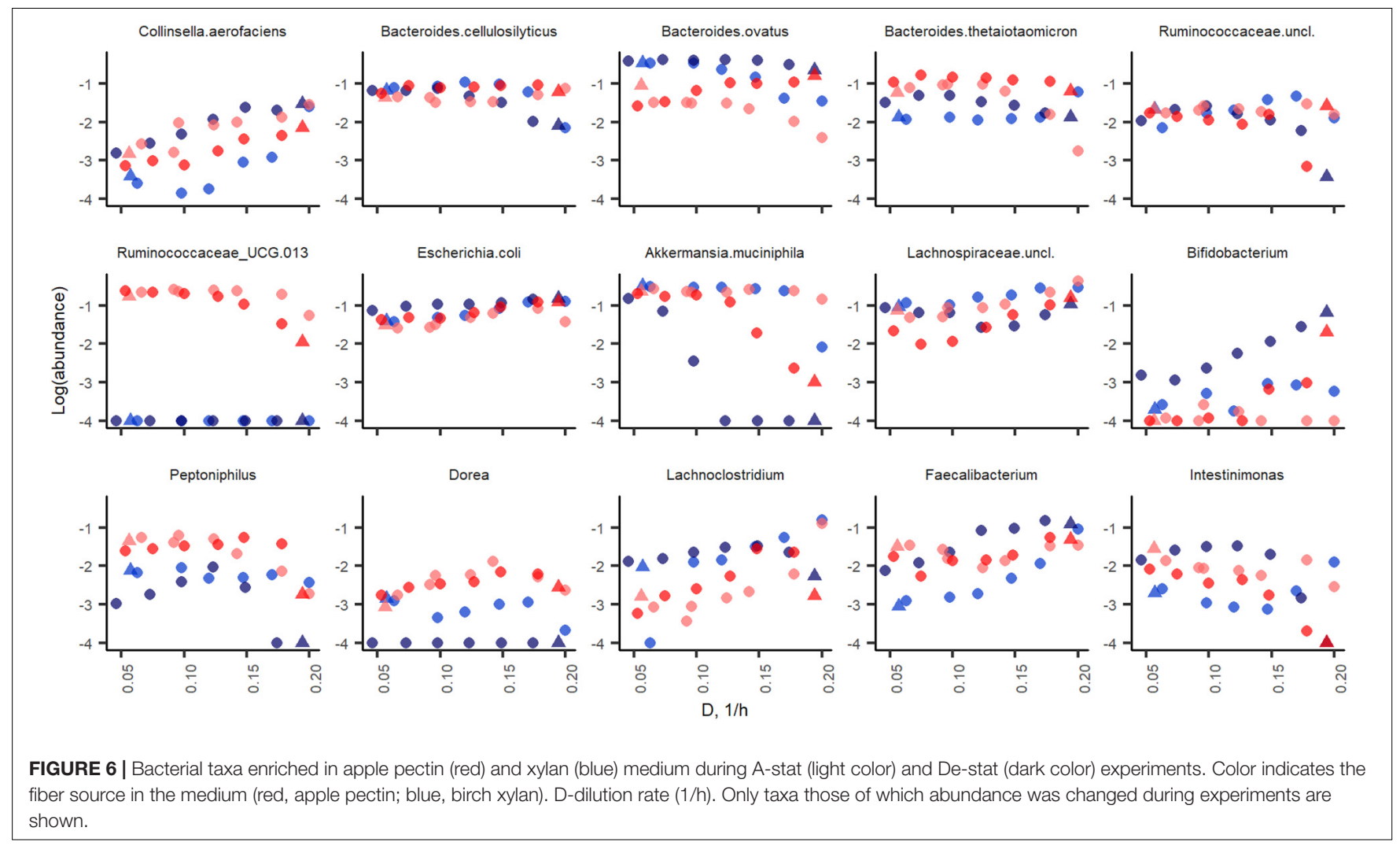

the following culture characteristics. Therefore, the microbiota and metabolite patterns were comparatively analyzed in A-stat and De-stat cultures in a defined base medium containing mucin and either apple pectin or xylan. Similar microbiota and metabolite structures were observed within the scanned range of dilution rates in both directions, from $D=0.05$ to $0.2 \mathrm{1} / \mathrm{h}$ or vice versa. The data of the steady state point of the chemostat at $D=0.2 \mathrm{1} / \mathrm{h}$ and the end point of the A-stat $(D=0.21 / \mathrm{h})$ coincided well. Thus, the fecal culture was able to adapt to the change rate applied. This shows that by using suitable acceleration or deceleration rates it is possible to achieve a state of culture comparable to those of classical chemostat cultures. This is new information for consortia cultivation, although it has long been known for pure cultures.

In a previous De-stat study with fecal samples from children (5-15 years old), similar structural changes in the fecal microbiota were seen (Adamberg and Adamberg, 2018). This suggests that for this age group adult-like microbiota are mostly established. Moreover, these data indicate the crucial role of the growth rate in metabolism and the structure of colon microbiota. For example, in both studies, the taxa clearly preferring high dilution rates were C. aerofaciens, Bifidobacterium, B. vulgatus, E. coli, Lachnospira and Lachnoclostridium, whereas A. muciniphila and the Ruminococcaceae group UCG-013 were enriched at low dilution rates. Accordingly, in vivo studies have shown that Akkermansia and ruminococci are more prevalent in people with slow colonic transit, while Bifidobacterium and Lachnospiraceae correlate with high transit rates (Kim et al., 2015; Roager et al., 2016; Vandeputte et al., 2016). The impact of pectin structure on the dynamics metabolism and fecal microbiota has been shown by Larsen et al. (2019). Highly methoxylated pectins were shown to stimulate $F$. prausnitzii, commonly referred to as a health-promoting species. In both of our studies with apple pectin, the abundance of Faecalibacterium was 1-7\% of the total population within the whole range of dilution rates, indicating the importance of pectin for the growth of colonic Faecalibacterium (the current study, and Adamberg and Adamberg, 2018).

Similarities between child and adult fecal pools were also observed at the metabolic level, but with some minor differences. Although the abundances of Faecalibacterium in adult and child cultures were similar (1.9-5.2\% and $4.5-7.5 \%$, respectively), about twice as much butyrate and $\mathrm{CO}_{2}$ were produced by adults' than by children's consortia at slow dilution rates, suggesting higher activity of the butyrate producers in adult microbiota. The dynamics of other metabolites, including BCFA and $\mathrm{H}_{2} \mathrm{~S}$, from the degradation of amino acids was comparable in both fecal consortia. The enhanced production of propionate, as well as the extensive use of amino acids and BCFA formation at low specific growth rates, may be related to a shortage of energy, ammonia, or $\mathrm{NAD}^{+}$regeneration. These properties are known for pure cultures (Tempest, 1984) but seem to also be common for fecal microbial consortia (Adamberg and Adamberg, 2018). $\mathrm{K}_{\mathrm{s}}$ values of carbohydrates and amino acids 
for different species should be measured to determine the carbon or nitrogen limitation, but these data are very scarce for gut bacteria.

The application of changestat makes it possible to elucidate the mechanisms of the co-existence of different bacteria by adapting mixed cultures under different environmental conditions. The cultivation of microbial consortia instead of single cultures is a promising approach for several biotechnological applications, including the development of multi-strain probiotics or material for fecal transplantation. As mentioned above, this and previous studies reveal that various consortia can be generated using continuous cultivation strategies. Still, the question remains of how to produce safe consortia with desired properties and/or therapeutic effects. Thus, detailed information is needed about selective pressure on the development of bacterial consortia under various environmental conditions: $\mathrm{pH}$, the availability and concentration of substrate, dilution rate, selective additives, defining the inocula, etc. The changestat approach makes it possible to scan selected environmental conditions in an adaptive manner; providing an opportunity to predict appropriate conditions for the development of a consortium with a desired bacterial pattern.

\section{CONCLUSION}

The changestat experiments presented in this paper showed that continuous cultures of complex fecal consortia are reproducible in chemostat. Similar microbiota and metabolite changes were observed within the scanned range of dilution rates in changestat cultures in both directions, from $D=0.05$ to $0.21 /$ h or vice versa. This is new information for consortia cultivation, although it has long been known for pure cultures.

Our work confirmed that dilution rate is a crucial trigger in consortia development. Some species, such as propionateproducing $B$. ovatus and $B$. vulgatus and butyrate-producing Faecalibacterium, were prevalent within the whole range of dilution rates, while the mucin-degrading bacterium A. muciniphila and some ruminococci were enriched at low dilution rates only.

The production of organic acids and gases from pectin in the presence of mucin was related to the dilution rate in chemostat cultures. The ratio of acetate, propionate and butyrate was $5: 2: 1$ at $D=0.51 / \mathrm{h}$ and $14: 2: 1$ at $D=0.21 / \mathrm{h}$. Most amino acids were completely depleted from the medium except for alanine and BCAA, which were metabolized to isobutyric and isovaleric acids in chemostat as well as changestat cultures.

For further analysis of the interactions in complex consortia, other gut-relevant environmental conditions and substrates available in the colon will be studied. It should be stressed that, in addition to high-throughput sequencing analysis, it is necessary to concentrate on the growth and metabolism of fecal consortia to work out novel methods for bacterial therapies. Changestat cultures make it possible to screen the combined effects of important environmental and feed parameters, such as acidity, temperature, medium composition, dilution rate, and the effects of inocula and multiple substrates.

\section{DATA AVAILABILITY STATEMENT}

The datasets analyzed for this study can be found in the European Nucleotide Archive on the site PRJEB33931 (ERP116764, dataset name "Run26_cult2").

\section{ETHICS STATEMENT}

The studies involving human participants were reviewed and approved by the Tallinn Medical Research Ethics Committee, Estonia (protocol No. 554). The patients/participants provided their written informed consent to participate in this study.

\section{AUTHOR CONTRIBUTIONS}

KA and SA designed the study. KA, GR, and SA carried out the experiments and data analyses. KA drafted the first manuscript. GR and SA contributed by writing and editing the final manuscript. All authors approved the submitted version.

\section{FUNDING}

This work was supported by the Estonian Ministry of Science and Education, Project number IUT1927.

\section{ACKNOWLEDGMENTS}

We would like to thank the Estonian Ministry of Science and Education for their funding. We are grateful to Betti Nigul for help in performing the growth experiments and Madis Jaagura for carrying out the bioinformatic analyses.

\section{SUPPLEMENTARY MATERIAL}

The Supplementary Material for this article can be found online at: https://www.frontiersin.org/articles/10.3389/fbioe.2020. 00024/full\#supplementary-material

FIGURE S1 | On-line measured data that are used for stability analyses during chemostat before A-stat (left side figures) or De-stat (right side figures) experiments. Upper figures show data in xylan + mucin medium and lower figures in apple pectin medium. Base rate, titration rate of $1 \mathrm{M} \mathrm{NaOH}(\mathrm{ml} / \mathrm{min}) ; \mathrm{rGas}$, gas production rate $(\mathrm{ml} / \mathrm{min}) ; \mathrm{D}$, dilution rate $(1 / \mathrm{h})$.

TABLE S1 | Abundances of bacteria in the inocula and samples from A-stat and De-stat experiments.

TABLE S2 | Production of metabolites per carbohydrate consumed ( $\mathrm{mol} / \mathrm{mol}$-carbohydrates and mmol/gDW), carbon recovery, and biomass yield (gDW/g-carbohydrates) during A-stat and De-stat experiments. 


\section{REFERENCES}

Adamberg, K., and Adamberg, S. (2018). Selection of fast and slow growing bacteria from fecal microbiota using continuous culture with changing dilution rate. Microb. Ecol. Health Dis. 29:1549922. doi: 10.1080/16512235.2018.154 9922

Adamberg, K., Kolk, K., Jaagura, M., Vilu, R., and Adamberg, S. (2018). The composition and metabolism of faecal microbiota is specifically modulated by different dietary polysaccharides and mucin: an isothermal microcalorimetry study. Benef. Microbes 9, 21-34. doi: 10.3920/BM2016.0198

Adamberg, K., Seiman, A., and Vilu, R. (2012). Increased biomass yield of Lactococcus lactis by reduced overconsumption of amino acids and increased catalytic activities of enzymes. PLoS One. 7:e48223. doi: 10.1371/journal.pone. 0048223

Adamberg, K., Valgepea, K., and Vilu, R. (2015). Advanced continuous cultivation methods for systems microbiology. Microbiolgy 161, 1707-1719. doi: 10.1099/ mic. 0.000146

Aguirre, M., Eck, A., Koenen, M. E., Savelkoul, P. H. M., Budding, A. E., and Venema, K. (2015). Evaluation of an optimal preparation of human standardized fecal inocula for in vitro fermentation studies. J. Microbiol. Methods 117, 78-84. doi: 10.1016/j.mimet.2015.07.019

Brahma, S., Martínez, I., Walter, J., Clarke, J., Gonzalez, T., Menon, R., et al. (2017). Impact of dietary pattern of the fecal donor on in vitro fermentation properties of whole grains and brans. J. Funct. Foods 29, 281-289. doi: 10.1016/j.jff.2016. 12.042

Burkitt, D. P., Walker, A. R. P., and Painter, N. S. (1972). Effect of dietary fibre on stools and transit-times, and its role in the causation of disease. Lancet 30, 1408-1411. doi: 10.1016/s0140-6736(72)92974-1

Bussolo de Souza, C., Jonathan, M., Saad, S. M. I., Schols, H. A., and Venema, K. (2019). Degradation of fibres from fruit by-products allows selective modulation of the gut bacteria in an in vitro model of the proximal colon. J. Funct. Foods 57, 275-285. doi: 10.1016/j.jff.2019.04.026

Chung, W. S. F., Walker, A. W., Louis, P., Parkhill, J., Vermeiren, J., Bosscher, D., et al. (2016). Modulation of the human gut microbiota by dietary fibres occurs at the species level. BMC Biol. 14:3. doi: 10.1186/s12915-015-0224-3

Chung, W. S. F., Walker, A. W., Vermeiren, J., Sheridan, P. O., Bosscher, D., GarciaCampayo, V., et al. (2019). Impact of carbohydrate substrate complexity on the diversity of the human colonic microbiota. FEMS Microbiol. Ecol. 95:fiy201. doi: 10.1093/femsec/fiy201

Cummings, J. H., Jenkins, D. J. A., and Wiggins, H. S. (1976). Measurement of the mean transit time of dietary residue through human gut. Gut 17, 210-218. doi: 10.1136/gut.17.3.210

Fallingborg, J., Christensen, L. A., Ingeman-Nielsen, M., Jacobsen, B. A., Abildgaard, K., and Rasmussen, H. H. (1989). PH-profile and regional transit times of the normal gut measured by a radiotelemetry device. Aliment. Pharmacol. Ther. 3, 605-613.

Freter, R., Stauffer, E., Cleven, D., Holdeman, L., and Moore, W. E. C. (1983). Continuous-flow cultures as in vitro models of the ecology of large intestinal flora. Infect. Immun. 39, 666-675. doi: 10.1128/iai.39.2.666-675.1983

Gottschal, J. C. (1990). Different types of continuous culture in -ecological studies. Methods Microbiol. 22, 87-124. doi: 10.1016/s0580-9517(08) 70240-x

Jorup-Rönström, C., Håkanson, A., Sandell, S., Edvinsson, O., Midtvedt, T., Persson, A. K., et al. (2012). Fecal transplant against relapsing Clostridium difficile-associated diarrhea in 32 patients. Scand. J. Gastroenterol. 47, 548-552. doi: 10.3109/00365521.2012.672587

Kasemets, K., Drews, M., Nisamedtinov, I., Adamberg, K., and Paalme, T. (2003). Modification of a-stat for the characterization of microorganisms. J. Microbiol. Methods 55, 187-200. doi: 10.1016/S0167-7012(03)00143-X

Kim, S. E., Choi, S. C., Park, K. S., Park, M. I., Shin, J. E., Lee, T. H., et al. (2015). Change of fecal flora and effectiveness of the short-term VSL\#3 probiotic treatment in patients with functional constipation. J. Neurogastroenterol. Motil. 21, 111-120. doi: 10.5056/jnm14048

Klindworth, A., Pruesse, E., Schweer, T., Peplies, J., Quast, C., Horn, M., et al., (2013). Evaluation of general 16 S ribosomal RNA gene PCR primers for classical and next-generation sequencing-based diversity studies. Nucleic Acids Res. 41, 1-11. doi: 10.1093/nar/gks808
Lagier, J.-C., Armougom, F., Million, M., Hugon, P., Pagnier, I., Robert, C., et al. (2012). Microbial culturomics: paradigm shift in the human gut microbiome study. Clin. Microbiol. Infect. 18, 1185-1193. doi: 10.1111/1469-0691. 12023

Langendijk, P., Schut, F., Jansen, G., Raangs, G., Kamphuis, G., Wilkinson, M., et al., (1995). Quantitative fluorescence in situ hybridization of Bifidobacterium spp. with genus-specific $16 \mathrm{~S}$ rRNA-targeted probes and its application in fecal samples. Appl. Environ. Microbiol. 61, 3069-3075.

Larsen, N., Bussolo de Souza, C., Krych, L., Cahú, T. B., Wiese, M., Kot, W., et al. (2019). Potential of pectins to beneficially modulate the gut microbiota depends on their structural properties. Front. Microbiol. 10:223. doi: 10.3389/fmicb.2019. 00223

Macfarlane, S., Quigley, M. E., Hopkins, M. J., Newton, D. F., and Macfarlane, G. T. (1998). Polysaccharide degradation by human intestinal bacteria during growth under multi-substrate limiting conditions in a three-stage continuous culture system. FEMS Microbiol. Ecol. 26, 231-243. doi: 10.1016/S0168-6496(98)000397

McDonald, J. A. K., Schroeter, K., Fuentes, S., Heikamp-deJong, I., Khursigara, C. M., de Vos, W. M., et al. (2013). Evaluation of microbial community reproducibility, stability and composition in a human distal gut chemostat model. J. Microbiol. Methods 95, 167-174. doi: 10.1016/j.mimet.2013. 08.008

Miller, T. L., and Wolin, M. J. (1981). Fermentation by the human large intestine microbial community in an in vitro semicontinuous culture system. Appl. Environ. Microbiol. 42, 400-407. doi: 10.1128/aem.42.3.400-407.1981

Paalme, T., Kahru, A., Elken, R., Vanatalu, K., Tiisma, K., and Vilu, R. (1995). The computer-controlled continuous culture of Escherichia Coli with smooth change of dilution rate (A-Stat). J. Microbiol. Methods 24, 145-153. doi: 10. 1016/0167-7012(95)00064-x

Petrof, E. O., Gloor, G. B., Vanner, S. J., Weese, S. J., Carter, D., Daigneault, M. C., et al. (2013). Stool substitute transplant therapy for the eradication of clostridium difficile infection: 'RePOOPulating' the gut. Microbiome 1:3. doi: 10.1186/2049-2618-1-3

Rajilić-Stojanović, M., Heilig, H. G. H. J., Molenaar, D., Kajander, K., Surakka, A., Smidt, H., et al. (2009). Development and application of the human intestinal tract chip, a phylogenetic microarray: analysis of universally conserved phylotypes in the abundant microbiota of young and elderly adults. Environ. Microbiol. 11, 1736-1751. doi: 10.1111/j.1462-2920.2009.01900.x

Roager, H. M., Hansen, L. B. S., Bahl, M. I., Frandsen, H. L., Carvalho, V., Gøbel, R. J., et al. (2016). Colonic transit time is related to bacterial metabolism and mucosal turnover in the gut. Nat. Microbiol. 1:16093. doi: 10.1038/nmicrobiol. 2016.93

Rowland, I., Gibson, G., Heinken, A., Scott, K., Swann, J., Thiele, I., et al. (2018). Gut microbiota functions: metabolism of nutrients and other food components. Eur. J. Nutr. 57, 1-24. doi: 10.1007/s00394-017-1445-8

Russell, J. B., and Baldwin, R. L. (1979). Comparison of substrate affinities among several rumen bacteria: a possible determinant of rumen bacterial competition. Appl. Environ. Microbiol. 37, 531-536. doi: 10.1128/aem.37.3.531-536.1979

Sender, R., Fuchs, S., and Milo, R. (2016). Are we really vastly outnumbered? Revisiting the ratio of bacterial to host cells in humans. Cell 164, 337-340. doi: 10.1016/j.cell.2016.01.013

Sghir, A., Chow, J. M., and Mackie, R. I. (1998). Continuous culture selection of bifidobacteria and lactobacilli from human faecal samples using fructooligosaccharide as selective substrate. J. Appl. Microbiol. 85, 769-777. doi: 10.1111/j.1365-2672.1998.00590.x

Tannock, G. W. (2017). Understanding Bowel Bacteria. Hoboken, NJ: John Wiley \& Sons, Inc.

Tempest, D. (1984). The status of YATP and maintenance energy as biologically interpretable phenomena. Annu. Rev. Microbiol. 38, 459-486. doi: 10.1146/ annurev.micro.38.1.459

Valgepea, K., Adamberg, K., and Vilu, R. (2011). Decrease of energy spilling in Escherichia coli continuous cultures with rising specific growth rate and carbon wasting. BMC Syst. Biol. 5, 106.

Vandeputte, D., Falony, G., Vieira-Silva, S., Tito, R. Y., Joossens, M., and Raes, J. (2016). Stool consistency is strongly associated with gut microbiota richness and composition. Enterotypes and bacterial growth rates. Gut 65, 57-62. doi: 10.1136/gutjnl-2015-309618 
von Martels, J. Z. H., Sadabad, M. S., Bourgonje, A. R., Blokzijl, T., Dijkstra, G., Faber, K. N., et al. (2017). The role of gut microbiota in health and disease: in vitro modeling of host-microbe interactions at the aerobe-anaerobe interphase of the human gut. Anaerobe 44, 3-12. doi: 10.1016/j.anaerobe.2017. 01.001

Yen, S., McDonald, J. A. K., Schroeter, K., Oliphant, K., Sokolenko, S., Blondeel, E. J. M., et al. (2015). Metabolomic analysis of human fecal microbiota: a comparison of feces-derived communities and defined mixed communities. J. Proteome Res. 14, 1472-1482. doi: 10.1021/pr501 1247
Conflict of Interest: The authors declare that the research was conducted in the absence of any commercial or financial relationships that could be construed as a potential conflict of interest.

Copyright (C) 2020 Adamberg, Raba and Adamberg. This is an open-access article distributed under the terms of the Creative Commons Attribution License (CC BY). The use, distribution or reproduction in other forums is permitted, provided the original author(s) and the copyright owner(s) are credited and that the original publication in this journal is cited, in accordance with accepted academic practice. No use, distribution or reproduction is permitted which does not comply with these terms. 